V.2, N.1, 2018

DOI: $10.33947 / 2595-6264-v 2 n 1-3552$

\title{
A ESTRUTURAÇÃO DE PASSADAS DO ROLO PÉ DE CARNEIRO NO PROCESSO DE COMPACTAÇÃO DO SOLO EM RODOVIAS
}

\section{THE STRUCTURATION OF PASSES OF CARNEIRO FOOT ROLL IN THE PROCESS OF SOIL COMPACTION IN ROADS}

\author{
Marcos Timóteo Rodrigues de Sousa', Marisa Vianna Mesquita²
}

\section{RESUMO}

O "fecha", nome dado aos trabalhos em rodovias quando se trata das questões das "passadas dos rolos" de compactação, é uma etapa que depende de muitos fatores: empíricos, técnicos, normativos e de experiência dos profissionais. O presente artigo tem por objetivo mostrar uma das etapas da metodologia de compactação do solo realizado em uma obra para pavimentação de rodovia. A quantidade de passadas depende de uma série de variáveis como: tipo de solo, sua densidade, grau de umidade e da energia exercida. Outros fatores preponderantes são os equipamentos utilizados, relacionados ao seu peso normal e o uso do lastro. Geralmente o grau de compactação aumenta já nas primeiras passadas do rolo de compactação, onde as passadas a mais podem prejudicar a compactação final do solo. Metodologias de cálculos como o método de "passadas" e os ensaios de compactação realizados em laboratórios como o de Proctor são de suma importância para uma compactação bem-sucedida.

PALAVRAS-CHAVES: Compactação do Solo. Rolo Pé de Carneiro. Rodovias.

\begin{abstract}
The "closure", the name given to the work on highways when it comes to the issues of the "roller coasters" of compaction, is a stage that depends on many factors: empirical, technical, normative and experience of the professionals. The present article aims to show one of the steps of soil compaction methodology carried out in a road-paving project. The number of passes depends on a series of variables such as: soil type, its density, degree of humidity and energy exerted. Other factors are the equipment used, related to its normal weight and the use of ballast. Generally, the degree of compaction increases in the first few passes of the compacting roller, where the extra passes may impair the final compaction of the soil. Calculation methodologies such as the "pass" method and the compaction tests carried out in laboratories such as Proctor's are of paramount importance for successful compaction.
\end{abstract}

KEYWORDS: Soil Compaction. Raisin Foot Roller. Highways. 
A ESTRUTURAÇÃO DE PASSADAS DO ROLO PÉ DE CARNEIRO NO PROCESSO DE COMPACTAÇÃO DO SOLO EM RODOVIAS Marcos Timóteo Rodrigues de Sousa, Marisa Vianna Mesquita

\section{V.2, N.1, 2018}

\section{INTRODUÇÃO}

Tanto no processo de ensino, em termos acadêmicos, quanto no cotidiano profissional, o entendimento dos métodos de análises são muito importantes para a organização de conceitos e dados quantitativos. Os trabalhos no qual envolvem os estudos do solo e os processos de pavimentação se complementam quando surge a necessidade da compactação do solo. Nos cursos de Engenharia Civil, Arquitetura, Geologia e tantos outros, os estudantes buscam os alicerces em referências bibliográficas, em experimentos laboratoriais e em dados empíricos para solucionar a questão da compactação do solo em campo.

Dentro de todos os métodos utilizados para essa atividade de compactação do solo, os rolos pé de carneiro e liso são um dos equipamentos que oferecem as meIhores tecnologias para a nossa realidade de compactação para pavimentação.

\section{COMPACTAÇÃO DO SOLO}

Segundo Kormann (2008) a AASHTO (American Association of State Highway and Transportation Officials), desde 1914, ano de sua fundação, tem a missão de estruturar modelos e métodos de análises para os estudos das Rodovias. Nogami (1983), cita que os métodos de análises e as técnicas da Geotecnia foram organizadas em solos não-tropicais, portanto, tal fato possibilitou pós anos de 1980, a produção de estudos dos solos em território brasileiro com a missão de entender os solos tropicais.

A questão do teor de umidade, tratada por Kormann (2008) é um fator preponderante nas relações entre os solos e o pavimento, pois, em rodovias de climas frios e temperados, os subleitos possuem uma grande proximidade com os níveis de águas e detém alto grau de saturação. Ainda segundo o autor, no Brasil os estudos tendem a aprimorar os métodos para compactar os solos de base e de subleito, tais estudos são realizados e analisados em laboratórios e no campo.

Já Caputo (2012), chama a atenção para a questão do fenômeno "Colapso", ou seja, a diminuição brusca do volume seco, quando encharcados e, sua deformação, relacionados à plasticidade e à liquidez. $O$ autor ainda cita que a presença frequente de espessas camadas naturais porosas com baixa densidade aparente é um fator complicador e, também cita sobre as ocorrências de variações volumétricas devido à alta concentração de umidades.

Segundo Santos (2002), diz que a compactação dos solos é a densificação do solo por meio mecânico, tal método traz a estabilização e melhoria, possibilitando a redução dos volumes vazios. Com este procedimento, e de forma adequada, os subleitos podem obter elevada capacidade de suporte.

\section{ROLO PÉ DE CARNEIRO}

De acordo com a ABNT NBR 15.115 (2004), relata que os equipamentos utilizados para a compactação em campo são os tratores de compactação e terraplanagem dotados de rolos com pequenos degraus em sua estrutura que garantem uma precisa compactação, podendo ser vibratórios ou não.

Um desses rolos, conhecido como pé de carneiro (figura 1), compacta de baixo para cima penetrando a camada solta superior e compactando a camada inferior, ou seja, quando o pé sai do solo ele joga para cima o material e o resultado é uma camada de aterial solto na porção superior e na inferior o solo compactado.

Figura 1: Rolo Pé de Carneiro em trabalho de compactação do solo em Rodovia.

Figure 1: Carneiro foot roll in soil compaction work on roads.

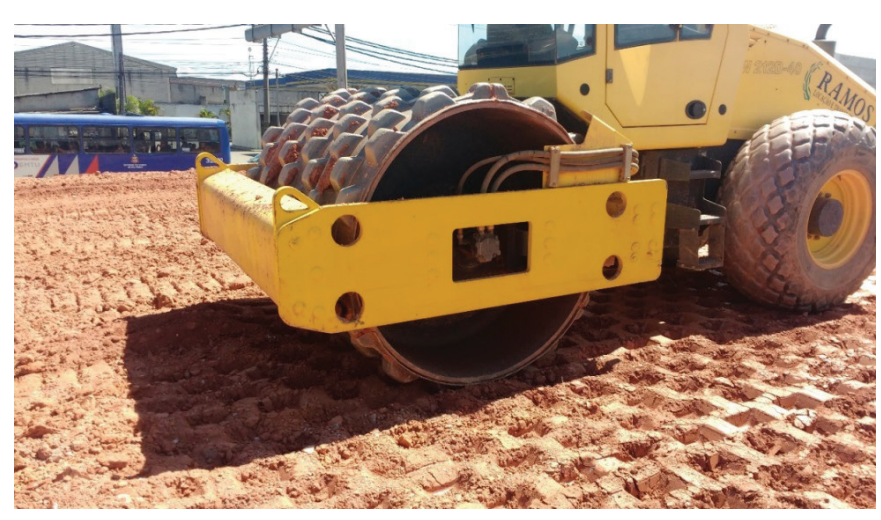

Foto: SOUZA (2018)

De acordo com Dias Júnior e Pierce (1996), a compactação é um método de estabilização de solos que se dá por aplicação de alguma forma de energia (impacto, vibração, compressão estática-amassamento ou dinâmica-vibratória). Seu efeito confere ao solo um aumento de seu peso específico e resistência ao cisaIhamento (deformação), e uma diminuição do índice de vazios, permeabilidade e compressibilidade que podem ser diagnosticados e analisados em laboratório e comprovados no campo. 
Segundo Caputo (2012), os trabalhos em laboratório, realizados por meio de ensaios de compactação resultam na correlação entre o teor de umidade e o peso específico seco de um solo quando compactado por uma determinada energia. O ensaio mais comum é o de Proctor, que é realizado através de sucessivos impactos de um soquete padronizado como mostram as figuras 2 e 3 .

Figura 2: Ensaio de compactação Proctor.

Figura 3: Resultado de compactação Proctor.

Figure 2: Proctor compaction test.

Figure 3: Proctor compaction result.

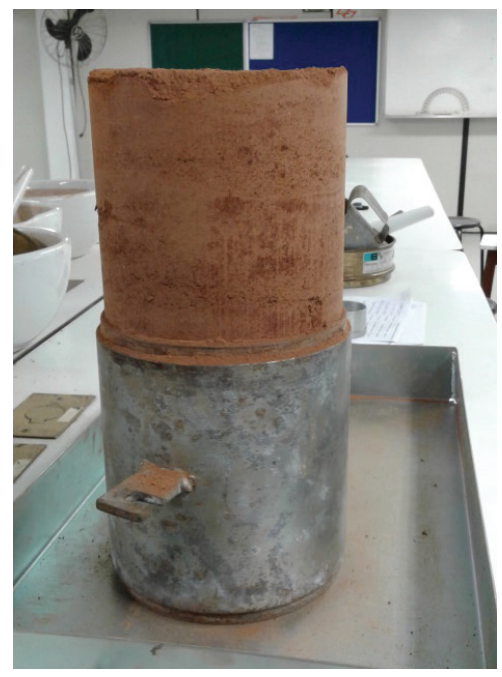

Foto: MESQUITA (2018)

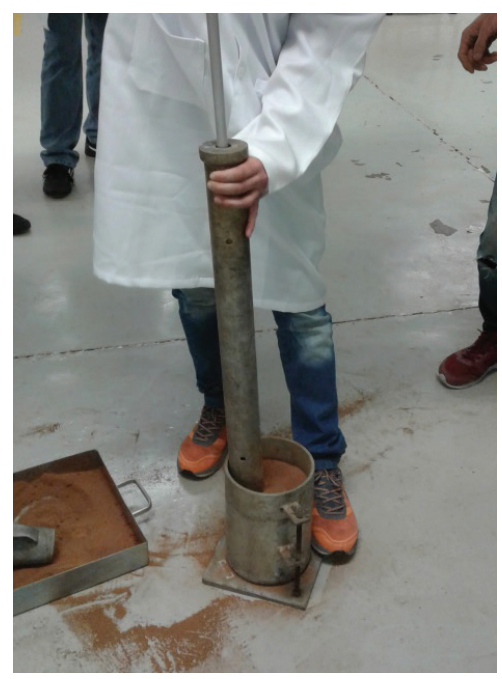

Foto: MESQUITA (2018)

De acordo com Kormann (2008), diz que há a necessidade da umidificação do solo em campo, sendo perceptível constatar que a adição de água no solo seco facilita a sua e muito sua compactação. Cada vez que se adiciona a água a esse solo pouco úmido, a densidade final do material compactado aumenta.

É comprovado que o acréscimo de água tem um efeito benéfico enquanto não se alcança um certo teor de umidade denominada de "umidade ótima". É de extrema importância determinar a umidade ótima do solo para uma dada energia de compactação e, também determinar o peso específico aparente seco máximo associado à umidade ótima. O peso máximo seco por umidade ótima gera o entendimento da resistência máxima do solo, ou seja, a expansão umidade/peso.

\section{MÉTODO DE "PASSADAS" DOS EQUIPAMENTOS}

Segundo Batista (1978), traz de forma bem didática a metodologia dos cálculos das "passadas" que os rolos pé de carneiro e liso realizam em campo. Segundo o autor, as "passadas" são os percursos em determinada área de largura igual à largura do rolo pé de carneiro e liso. $O$ rolo pé de carneiro tem um princípio efetivo de compactação enquanto o rolo liso gera o acabamento, trabalho este realizado em conjunto.

Utilizando-se da metodologia de Batista (1978) em campo, pode-se entender o processo de passadas como, por exemplo, a cobertura da área de contato da compactação se refere à largura da estrada em relação à largura do rolo.

Na figura 4 pode-se observar a ação do rolo fazendo o seu trabalho de compactação do solo. A quantidade de "passadas" é muito relativa, pois, depende do tipo do solo, da umidade específica, do tipo de equipamento utilizado e as especificações das Normas do DNIT (Departamento Nacional de Infraestrutura de Transporte). O acompanhamento do controle tecnológico é de extrema importância, pois, são realizados os ensaios a cada trabalho a ser executado.

De acordo com Batista (1978) a fórmula básica das passadas é:

$\mathbf{P}=$ Passadas;

$$
\mathbf{P}=\mathbf{2} \times \pi \times \mathbf{R} \times \mathbf{I} / \mathbf{N} \times \mathbf{A} \text { onde: }
$$

$\mathbf{R}=$ Raio do tambor do rolo;

$\mathbf{L}=$ largura do rolo;

$\mathbf{N}=$ quantidade de passadas;

$A=$ cobertura de um pé do rolo.

Por meio desta fórmula o autor sugere a fragmentação 
A ESTRUTURAÇÃO DE PASSADAS DO ROLO PÉ DE CARNEIRO NO PROCESSO DE COMPACTAÇÃO DO SOLO EM RODOVIAS Marcos Timóteo Rodrigues de Sousa, Marisa Vianna Mesquita

\section{V.2, N.1, 2018}

dos cálculos, dissertando passo-a-passo cada etapa.

Figura 4: Processo de "passadas" do rolo em campo. Figure 4: Rolling process in the field.

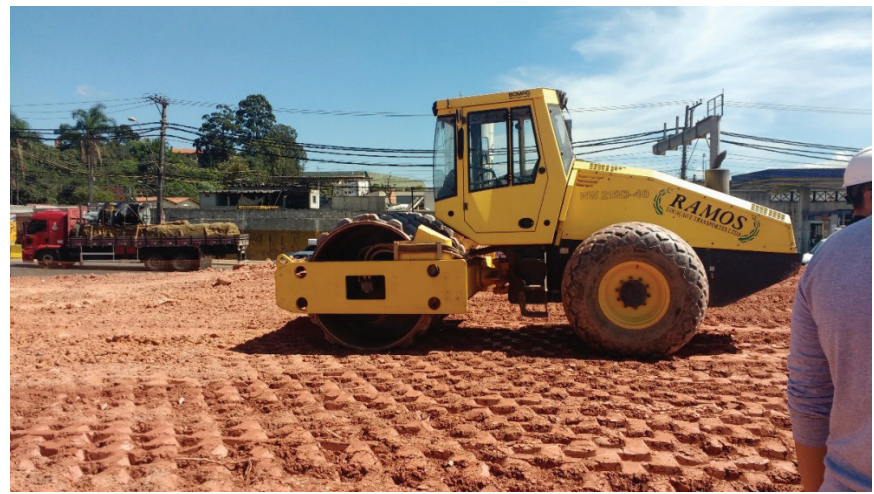

Foto: SOUZA (2018)

\section{APLICABILIDADE DO MÉTODO DE "PASSADAS" EM CAMPO}

A área escolhida para se utilizar o método de "passadas", foi nas obras do Trevo de Bonsucesso com a Rodovia Presidente Dutra (BR 116), localizada na cidade de Guarulhos, obra esta, em andamento a cerca de muitos anos.

A figura 5 ilustra uma das etapas da obra, no qual as estacas foram inseridas e as máquinas iniciaram o processo de compactação do solo para a futura inserção do revestimento.

A metodologia de cálculo utilizado foi de Batista (1978), como demonstrado a seguir:

\section{a) Área efetiva da passada do rolo}

\section{S $=2 \times \pi \times R \times I$ onde:}

\section{$\mathbf{S}=$ Área Efetiva da Passada do Rolo}

$\mathbf{R}=$ Raio (tambor do rolo) $=0,71 \mathrm{~m}$

I = Largura do Rolo $=1,22 \mathrm{~m}$

$$
\mathbf{S}=2 \times 3,1416 \times 0,71 \mathrm{~m} \times 1,22 \mathrm{~m}=\mathbf{5 , 4 3 9 7 \mathbf { m } ^ { 2 }}
$$

Por meio destas informações chega-se ao resultado de $\mathbf{5 , 4 3 9 7} \mathrm{m}^{2}$ a área efetiva da passada.
Figura 5: Processo de estaqueamento e compactação do solo.

Figure 5: Stacking process and soil compaction.

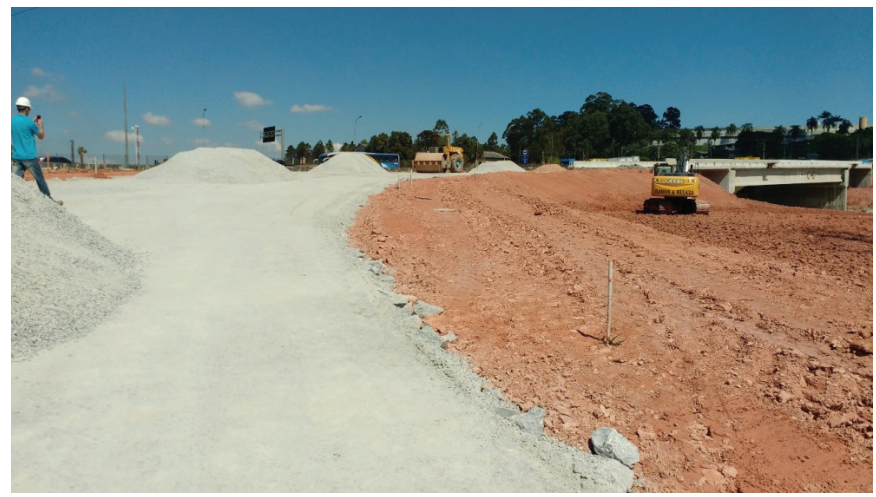

b) Cobertura de um pé do rolo

Foto: SOUZA (2018).

$$
\mathbf{A}=\mathbf{N} \times \mathbf{a} \text { onde: }
$$

$A=$ Cobertura de um pé do rolo

$\mathbf{N}=$ Quantidade de pés do rolo $=176$ pés

$a=$ Área de contato de um pé $=30 \mathrm{~cm}^{2}$

$$
A=176 \times 30=5,280 \mathrm{~cm}^{2}
$$

Por meio destas informações chega-se ao resultado de $5,280 \mathrm{~cm}^{2}$ a área de cada pé do rolo.

c) Quantidade de passadas efetivas

$$
\begin{aligned}
& \mathbf{C}=\mathbf{S} / \mathbf{A} \text { onde: } \\
& \mathbf{S}=\text { Área Efetiva }=5,4397 \mathrm{~m}^{2} \\
& \mathrm{~A}=\text { Cobertura de um Pé do Rolo }=5,280 \mathrm{~cm}^{2} \\
& \text { C }=5,4397 \mathrm{~m}^{2} / 5,280 \mathrm{~cm}^{2} \\
& C=5,4 / 0,52=10,40 \text { Passadas }
\end{aligned}
$$

Por meio destas informações chega-se ao resultado de 10,40 passadas em uma determinada área.

d) Fórmula básica das passadas

$$
\mathrm{P}=2 \times \pi \times \mathrm{R} \times \mathrm{I} / \mathrm{N} \times \mathrm{A}
$$$$
\mathbf{P}=2 \times 3,14 \times 0,71 \times 112 / 176 \times 30
$$

$P=10,40$ Passadas 
A ESTRUTURAÇÃO DE PASSADAS DO ROLO PÉ DE CARNEIRO NO PROCESSO DE COMPACTAÇÃO DO SOLO EM RODOVIAS Marcos Timóteo Rodrigues de Sousa, Marisa Vianna Mesquita

V.2, N.1, 2018

\section{CONSIDERAÇÕES FINAIS}

De acordo com o resultado do método de "passadas" (Batista, 1978), significa que na prática, o rolo pé de carneiro deve passar mais de 10 vezes no mesmo local para efetivar uma boa compactação do solo (de 10 a 20 passadas por área de cobertura), lembrando que esses números dependem de uma série de variáveis do local.

O cálculo é importantíssimo, pois um solo com uma "umidade ótima" de compactação pode aumentar por demais sua densidade caso tenha uma maior quantidade de passadas tornando a compactação inviável.

Outros fatores, de extrema importância que também interferem na passagem do rolo e que necessitam serem levados em consideração são: largura da faixa de rolamento; comprimento da via que determinará a produção total por hora; a velocidade do rolo (quanto mais lento maior a compactação e quanto mais profundo o subleito, menor a pressão de contato do rolo), nivelamento do terreno e tipo de solo, todas variáveis que deverão ser determinadas no momento da utilização do processo de compactação para que o resultado da obra e a colocação do revestimento para construção da rodovia sejam bem sucedidos.

\section{REFERÊNCIAS}

ABNT (ASSOCIAÇÃO BRASILEIRA DE NORMAS TÉCNICAS). Execução de camadas de pavimentação, NBR 15115. Rio de Janeiro: Comitê Brasileiro de Construção Civil, 2004.

BAPTISTA, C. N. Pavimentação. Porto Alegre: Globo, 1978.

CAPUTO, H. P. Mecânica dos solos e suas aplicações. Rio de Janeiro: Livros Técnicos e Científicos Editora S.A, 2012. 3 v.

DIAS JUNIOR, M. S, PIERCE, F. J. O processo de compactação do solo e sua modelagem. Revista Brasileira Ciências do Solo, v. 20. p. 175-182, 1996.

KORMANN. A. C. Ensaio de compactação e caracterização. Curitiba: UFPR, 2008.

NOGAMI, J. S. Os solos tropicais lateríticos e saprolíticos e a pavimentação. In: REUNIÃO ANUAL DE PAVIMENTAÇÃO, 18․ 1983. Porto Alegre. Anais... Porto Alegre, 1983.

SANTOS, A. R. Geologia de engenharia: conceitos, método e prática. São Paulo: IPT/ABGE, 2002. 